\title{
Using Liquid Crystal Assisted Replica Molding Method to Align Uniaxial Molecules in Patterned Polymers at Ambient Temperature
}

\author{
Pei-Shiang Chen ${ }^{1}$, Jun-Wei Chen ${ }^{1}$, Han-Hsun Chang ${ }^{1}$, Ching-Hsiang Hsu ${ }^{1}$, \\ Yuan-Huei Chang ${ }^{1}$, Hsiang-Lin Liu ${ }^{2}$, Chieh-Hsiung Kuan ${ }^{3}$ and Chih-Yu Chao ${ }^{1}$ \\ ${ }^{1}$ Department of Physics, National Taiwan University, Taipei 10617, Taiwan \\ ${ }^{2}$ Department of Physics, National Taiwan Normal University, Taipei 11677, Taiwan \\ ${ }^{3}$ Department of Electrical Engineering, National Taiwan University, Taipei 10617, Taiwan \\ E-mail: pschen@phys.ntu.edu.tw
}

\begin{abstract}
In this paper, we demonstrate an easy approach - liquid crystal assisted replica molding method - to align uniaxial molecules in a patterned polymer at ambient temperature. During the replica molding process, the orientation of the liquid crystals is parallel to the groove of the grating mold and the uniaxial molecules are aligned by liquid crystals. In order to confirm our observations, we have measured polarized absorption and photoluminescence (PL) spectra to quantify the degree of alignment. Because of the characteristic described above, this approach to align molecules in a polymer opens up an easy way to manufacture optoelectronic and electronic devices.
\end{abstract}

\section{Introduction}

The alignment of molecules by nanoconfinement is an important and interesting issue nowadays for the potential applications of aligned molecules. For example, molecular orientation in semiconducting polymers leads to anisotropic electronical conductivity [1]; molecular orientation in polymers leads to anisotropic optical devices such as optical polarizers; oriented molecules are used as an active layer in polymeric light-emitting diodes (PLED) to emit polarized light[2-4]; aligned molecules result in second-order nonlinear optical properties and these properties are frequently functioned in electro-optical modulator [5] and frequency converter [6].

There are several methods used to align molecules in a polymer: (1) stretching/rubbing polymer films or polyimide (PI) [3,7], (2) doping luminescent guest molecules in an aligned host matrix [8,9], (3) aligning molecules with magnetic, electric field, or photoirradiation [5,10-14], (4) Langumuir-Blodgett deposition (self-assembly) $[15,16]$. However, the problem of rubbing is that we can hardly control the direction of grooves and it can introduce dust and static charge into rubbing process; when stretching polymer films, we cannot control the deformation by elongation easily. Doping molecules in an aligned host matrix cannot have good alignment because the molecules do not have strong interactions with each other. We need very strong magnetic or electric field to align molecules well.

Langumuir-Blodgett deposition can be applied only to specific molecules such as molecules with hydrophilic and hydrophobic polar. Those methods above are either hard to control or limited to certain molecules.

In this paper, we demonstrate an easy approach to align molecules in the plane of polymer films at ambient temperature. Here are some of the advantages of this approach. The samples need not to undergo high temperature; even non-heat-resistant molecules will not be destroyed throughout the process; because it combines the aligning ability of liquid crystals and the advantages of replica molding method together [17], we can use it to align many kinds of uniaxial molecules. The alignment of molecules is observed in the Poly(dimethylsiloxane) (PDMS) films after we pattern them by replica molding method, an efficient and effective way to duplicate the surface structure of a mold. The benefits of replica molding method are: low fabrication costs, high-throughput, simple fabrication, and the ability to copy nanometer scale structures over many centimeters. In addition, the mold is reusable 
since the curing and peeling of the PDMS substrates does not destroy the original one. In other words, we can make many PDMS films by only one mold. Therefore, we can decrease the costs and time on producing molds. This liquid crystal assisted replica molding method not only saves the costs of manufacturing but also broadens the applications of molecules.

\section{Experimental}

In this work, we used liquid crystal assisted replica molding method to fabricate flexible devices. Figure 1 is the flowchart of the liquid crystal assisted replica molding method fabrication process we have used in this paper. The uniaxial molecules and liquid crystals are mixed with PDMS. The mixed PDMS is dropped on the silicon mold and deposited at ambient temperature. The liquid crystals are aligned by the grooves of the grating mold and the uniaxial molecules are aligned by the liquid crystals. After 24 hours, the mixed PDMS can be readily converted into solid elastomers by cross-linking. After it is cured, we peel PDMS against the rigid mold. The PDMS with aligned molecules is complete right afterwards.

(a)

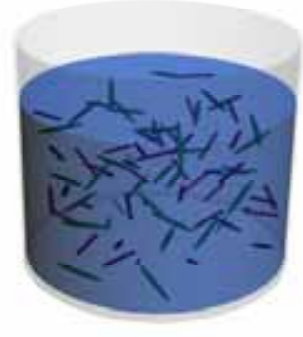

(c)

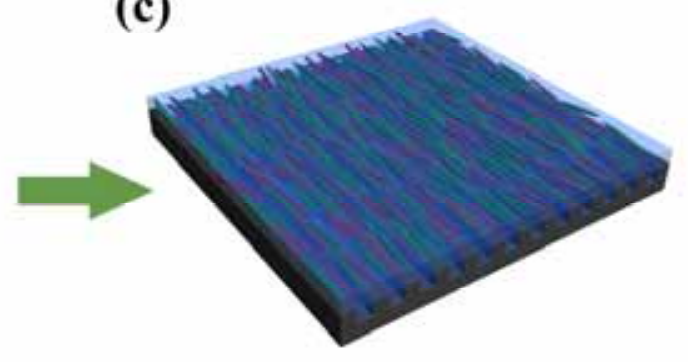

(e)

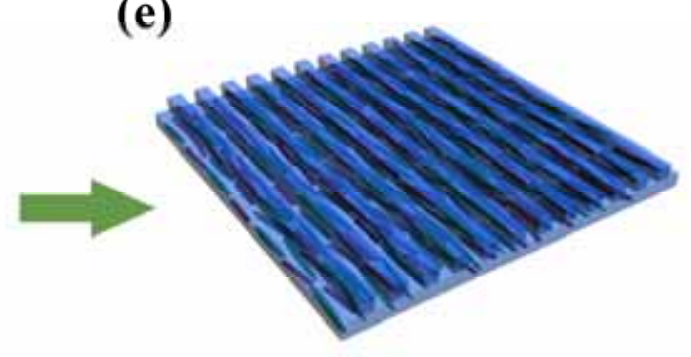

(b)

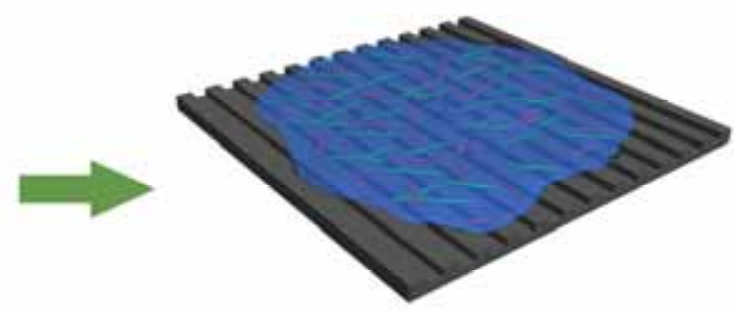

(d)

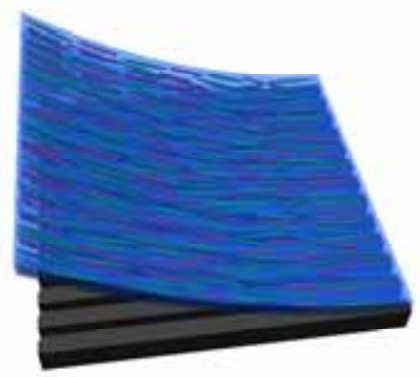

Figure 1. Flowchart of the fabrication process of PDMS/dye substrates with liquid crystals.

(a) Mixing dyes and liquid crystals with PDMS.

(b) Dropping mixed PDMS on the silicon mold.

(c) Depositing mixed PDMS at ambient temperature for 24 hours.

(d) Peeling mixed PDMS against mold.

(e) Flexible mixed PDMS substrate. 
During our research process, the mold is made up of silicon wafer with microgrooves patterned via traditional photo-lithography process. The pitch of the grating is $1 \mu \mathrm{m}$ and the line-width of the grating is $500 \mathrm{~nm}$. As for PDMS, it is commercially available from Dow Corning Corporation. It is supplied as a two-part kit: a liquid silicon rubber base and a curing agent. Here, we mix curing agent and liquid silicone rubber base at a ratio of 1:5. Since that the PDMS elastomer is isotropic and homogeneous with good thermal stability up to $186^{\circ} \mathrm{C}$, substrates made from this material can be deformed mechanically to fit the patterns in surfaces of modes. The formulation, applications, and fabrication of PDMS elastomers have been extensively studied in Reference [18].

The uniaxial molecules and liquid crystals are mixed with PDMS before PDMS is cured. We mix $0.02 \%$ wt uniaxial molecules with liquid crystals and PDMS by stirring. There are three kinds of uniaxial molecules offered by Exciton Corporation separately: DCMII, LDS698, and LD700. Figure 2a to 2c shows the molecule structures of DCMII, LDS698, and LD700. Those molecules are organic dye molecules and their dipoles are oriented mainly along the molecular chain direction. We measured the PL and optical absorption to make sure if the molecules have macroscopic anisotropic orientation. PL measurements were carried out at room temperature and the excitation source is a $325 \mathrm{~nm} H e-C d$ laser. In order to measure the luminescence of different polarization, an optical polarizer is inserted in front of the photomultiplier tube for us to select the signal polarization direction. The photomultiplier tube is used to enhance the signals and lock-in amplifier is used to lock the signals. Optical absorption was carried out at room temperature and an optical polarizer is inserted in front of the light source for us to produce light of different polarization. In order to analyze different wavelengths, we used spectroscope to measure the absorption.

(a)<smiles>N#CC(C#N)=C1C=COC(C=Cc2cc3c4c(c2)CCCN4CCC3)=C1</smiles>

(b)<smiles>CC[n+]1ccccc1C=CCc1ccc(N(C)C)cc1</smiles>

(c)<smiles>FC(F)(F)C1=c2cc3c4c(c2Oc2c1cc1c5c2CCCN5CCC1)CCC[N+]=4CCC3</smiles>

Figure 2. Molecule structures.
(a) DCMII.
(b) LDS698.
(c) LD700. 


\section{Results and discussion}

Figure 3 shows the results of samples with DCMII [FIG. 3(a)], LDS698 [FIG. 3(b)], and LD700 [FIG. $3(c)$ ], respectively. According to the results of figure 3, it is found that the luminescence intensity is strongest when the grating direction of the samples is parallel to the polarization direction of polarizer; the luminescence intensity is the weakest when the grating direction of the samples is perpendicular to the polarization direction of polarizer. The dichroism ratio for the DCMII, LDS698, and LD700 samples with liquid crystals are 2.02, 1.99, and 2.08, respectively. This result tells us that the mixed PDMS films have a very good anisotropic property because the dichroism is in proportion to the anisotropic degree. Therefore, we can find that liquid crystals have very good microscopically orientation along the grating direction, and the uniaxial dye molecules are oriented by liquid crystals. In order to confirm the result, we also measure optical absorption spectrum. In figure 4 , the mixed PDMS have a larger absorption when the polarized light parallels to the grating direction and the absorption difference is obvious. The measurement of optical absorption spectrum can also support the result. Therefore, measurement results of PL and optical absorption spectrum are consistent with each other. For comparison, we measured the polarized luminescence intensity and absorption of PDMS/DCMII without liquid crystals. In figure 5, there was no obvious anisotropic photoluminescence and optical absorption behavior observed. The uniaxial molecules are not aligned well without the assistance of liquid crystals and they are aligned randomly. From the macroscopic view, the PDMS film with only uniaxial molecules is an isotropic one. 
(a)

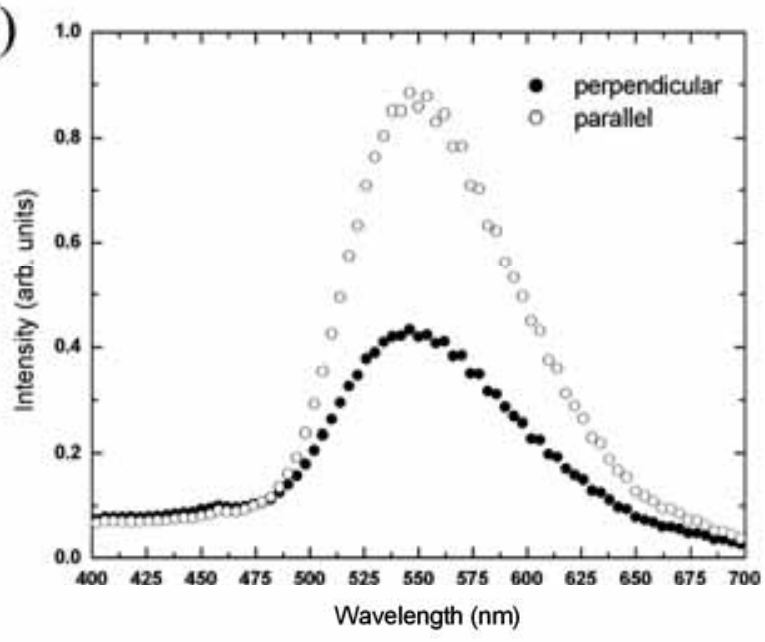

(b)

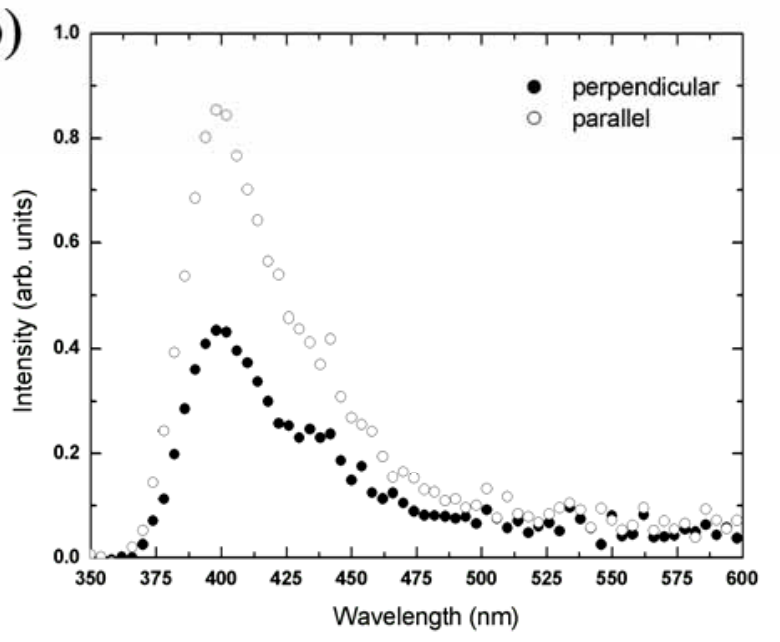

(c)

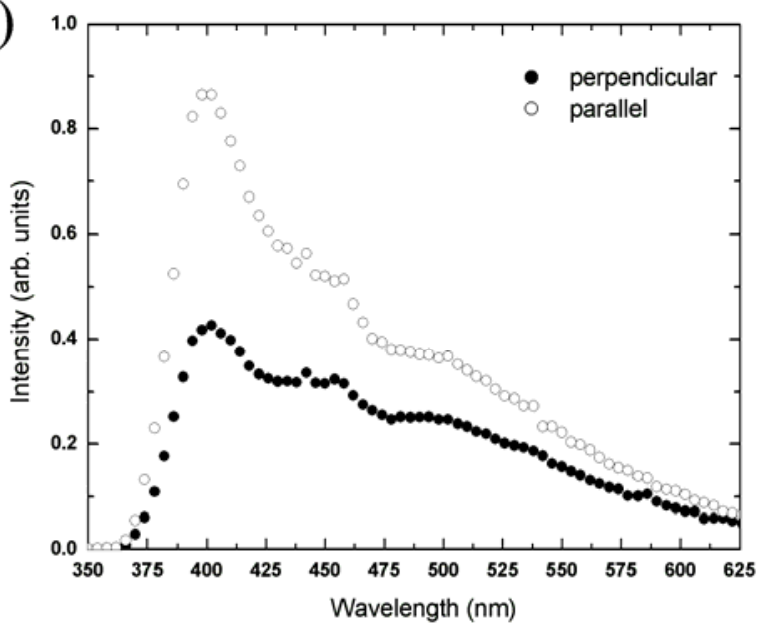

Figure 3. Polarized photoluminescence measurement results.

(a) PDMS/DCMII with liquid crystals.

(b) PDMS/LDS698 with liquid crystals.

(c) PDMS/LD700 with liquid crystals. 
(a)

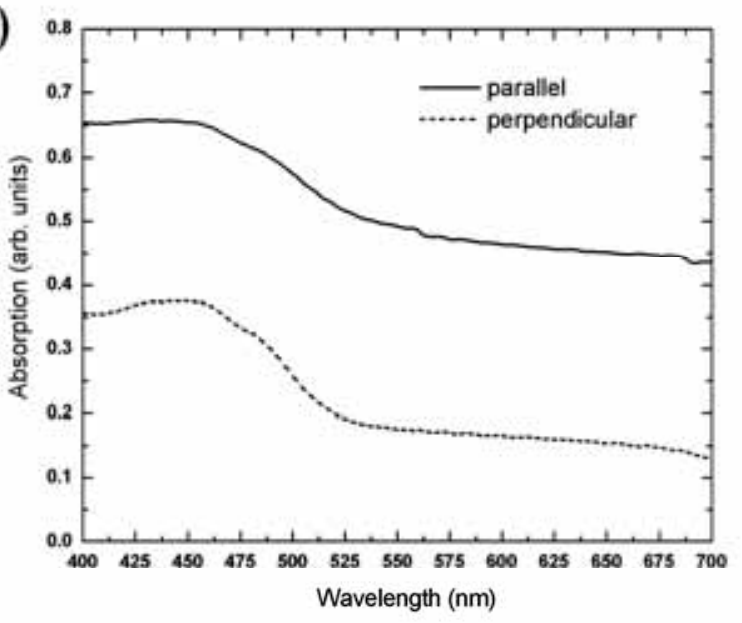

(b)

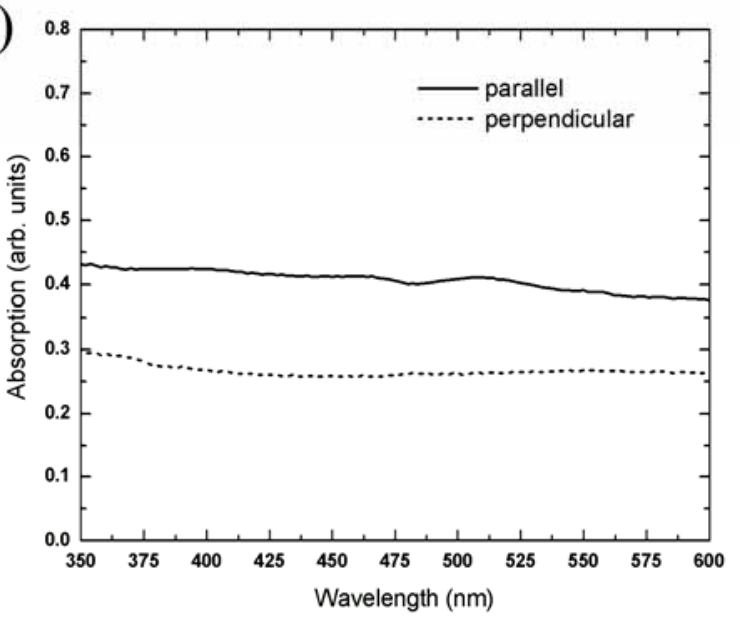

(c)

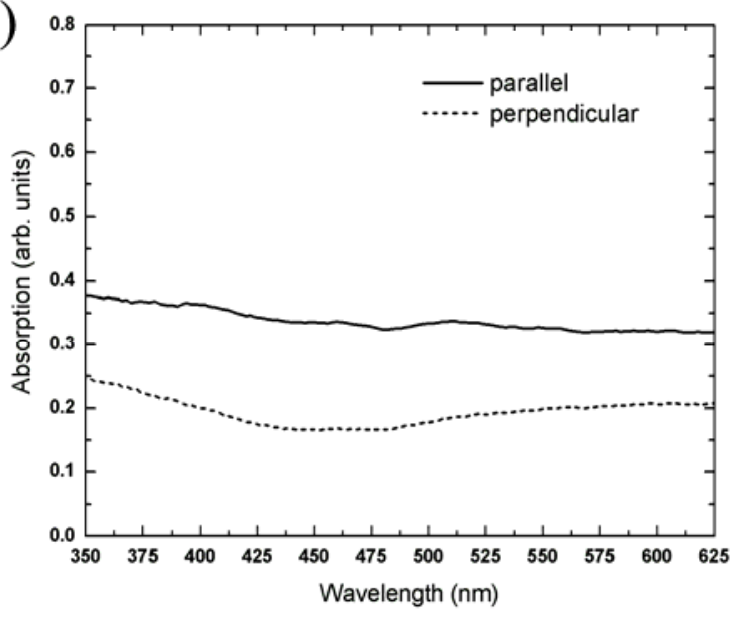

Figure 4. Polarized absorption measurement results.

(a) PDMS/DCMII with liquid crystals.

(b) PDMS/LDS698 with liquid crystals.

(c) PDMS/LD700 with liquid crystals. 

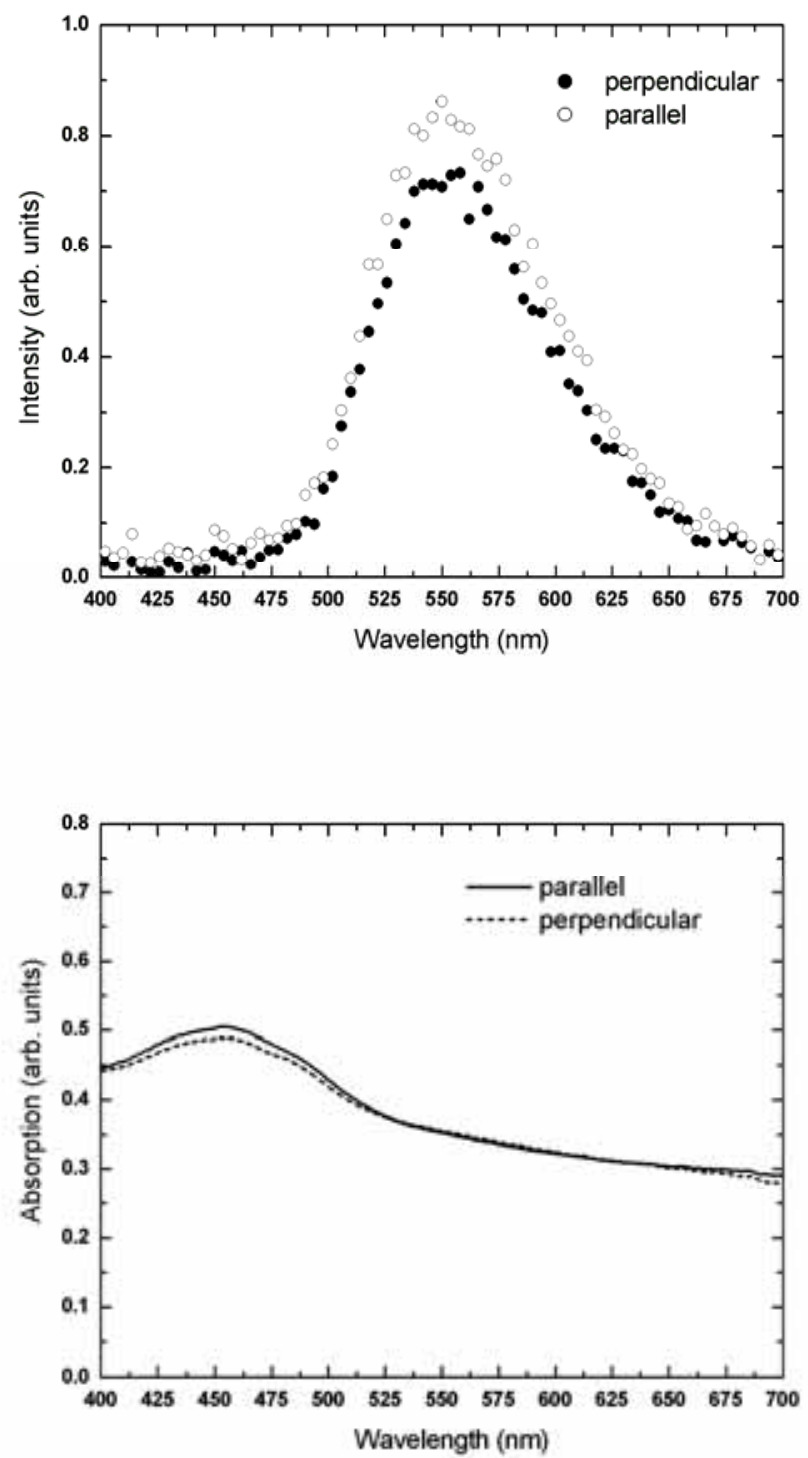

Figure 5. Polarized photoluminescence and absorption measurement results of PDMS/DCMII without liquid crystals.

\section{Conclusion}

In conclusion, liquid crystal assisted replica molding method with grating molds is a simple and inexpensive method when we align uniaxial molecules in a patterned polymer. With the assistance of liquid crystals, uniaxial molecules can be aligned well along the grooves of molds. Also, unlike nanoimprint method [19], this method does not need to function with high temperature. Therefore, it can be applied to more kinds of uniaxial molecules. Moreover, it can be extended to other polymers and molecules for further applications. To sum up, it is undoubtedly that this method will be an important application for optoelectronics, molecular electronics, and optical devices.

\section{Acknowledgment}

We thank the National Nano Device Laboratories for manufacturing the grating molds. This work was supported by the National Science Council and Ministry of Education of Taiwan. 


\section{References:}

[1] Dyreklev P and Inganas O 1994 J. Appl. Phys. 767915

[2] Grell M, Knoll W, Lupo D, Meisel A, Miteva T, Neher D, Nothofer H G, Scherf U and Yasuda A 1999 Adv. Mater. 11671

[3] Dyreklev P, Berggren M, Inganas O, Andersson M R, Wennerstrom O and Hjertberg T 1995 Adv. Mater. 7 43

[4] Era M, Tsutsui T and Saito S 1995 Appl. Phys. Lett. 672436

[5] Lee H, Hwang W, Oh M, Park H, Zyung T and Kim J 1997 Appl. Phys. Lett. 713779

[6] Burland D M, Miller R D, Reiser O, Twieg R J and Walsh C A 1992 J. Appl. Phys. 71410

[7] Hamaguchi M and Yoshino K 1995 Appl. Phys. Lett. 672281

[8] Hagler T W, Pakbaz K, Voss K F and Heeger A J 1991 Phys. Rev. B 448652

[9] Bastiaansen C, Schmidt H W, Nishino T and Smith P 1993 Polymer 343951

[10] Shklyarevskiy I O, Jonkheijm P, Stutzmann N, Wasserberg D, Wondergem H J, Chiristianen P C M, Schenning A P H, Leeuw D M, Tomovic Z, Wu J, Mullen K and Mann J C 2005 J. Am. Chem. Soc. 127 16233

[11] Xu Z S, Lemieux R P and Natansohn A 1998 Chem. Mater. 103269

[12] Jin S H, Seo H U, Nam D H, Shin W S, Choi J H, Yoon U C, Lee J W, Song J G, Shin D M and Gal Y S 2005 J. Mater. Chem. 155029

[13] Yitychaik S, Bella S D, Lundquist P M, Wong G K and Marks T J 1997 J. Am. Chem. Soc. 1192995

[14] Buffeteau T, Labarthet F L, Peyolet M and Sourisseau C 1998 Macromolecules 31, 7312

[15] Ma S, Yan M, Lu X, Wang W, Chen Z, Liu P, Qu C, Lin S and Ding A 1999 Appl. Phys. Lett. 742065

[16] Cimrova V, Remmers M, Neher D and Wegner G 1996 Adv. Mater. 8146

[17] Xia Y and Whitesides G M 1998 Annu. Rev. Mater. Sci. 28153

[18] Xia Y, McClelland J J, Gupta R, Qin D, Zhao X M, Sohn L L, Celoua R J and Whitesides G M 1997 Adv. Mater. 9147

[19] Chou S Y, Krauss P R and Renstrom P J 1996 J. Vac. Sci. Technol. B 144129 


\title{
Using Replica Molding Method to Fabricate Alignment-layer free Flexible Liquid Crystal Devices
}

Pei-Shiang Chen, Han-Hsun Chang, Jun-Wei Chen, Tzu-Chieh Lin and Chih-Yu Chao

\author{
Department of Physics, National Taiwan University, Taipei 10617, Taiwan \\ E-mail address: pschen@phys.ntu.edu.tw
}

\begin{abstract}
Flexible liquid crystal display (LCD) has become a popular topic nowadays. Many kinds of processes have been used to make flexible LCDs. In this paper, we fabricate a flexible LC device by replica molding method - using Poly(dimethylsiloxane) (PDMS) rather than plastic to be the substrate. Here, the PDMS acts not only as the substrate but also the alignment-layer in the flexible LCD; thus there is no need to coat additional alignment-layer on the flexible substrates. According to our results, we have successfully increased the flexibility of rollable LC devices via replica molding method. Also, fewer fabrication steps are needed as compared with other conventional processes. These two achievements can definitely increase the range of applications and reduce the cost of the manufacturing process.
\end{abstract}

\section{Keywords}

flexible LCD, replica molding 


\section{Introduction}

A lot of attention has been paid to flexible displays because they have many unique features such as lightness, thin packaging and portability. As for the substrates, although glass ones are often used in conventional LCDs, they are inflexible and fragile. Recently, glass substrates are commonly replaced by plastic or bendable substrates such as polyethylene terephthalate (PET) film because of flexibility and thinness. E-papers can be one of the most commonly known examples. At the same time, aligning liquid crystals (LCs) uniformly on plastic or bendable substrates becomes a crucial problem during flexible display manufacturing process. An available way to align LCs is coating polymer layer and then making microgrooves above. These kinds of methods include rubbing and nano-imprint lithography ${ }^{1,2)}$. However, since that the substrates coated with polymer layers are bended for many times, the cracks of the polymer layer will get bigger and bigger as time goes by. In order to solve this problem, we have tried to make the bendable materials serve not only as substrates but also as alignment-layers; naturally, how to achieve the above two goals all together is undoubtedly an important research focus in our research. If both objects are achieved, we can definitely lessen the steps in the process of fabricating flexible displays.

In this paper, we fabricate grating nanostructures directly on flexible substrates to align LCs by using replica molding method ${ }^{3)}$. We have chosen replica molding method for many reasons. First, replica molding method is an efficient way to duplicate the structure on the surface of a mold with the benefits of low fabrication costs, high-throughput, simple fabrication, and the capability to duplicate nanometer scale structures over many centimeters; since that curing and peeling PDMS substrates do not destroy the original mold, we can use the mold to make additional substrates by repeating the preceding steps; in the end, the cured polymers will possess almost the same dimensions and topologies of the mold; Second, it is replica molding method that allows us to duplicate three-dimensional topologies in a single step. Without it, we have to execute complicated steps such as exposure and etching when we conduct traditional photo lithography; in addition, it is rather difficult for us to make three-dimensional patterns via the classical method. Third, replica molding method lets us duplicate soft and flexible materials directly, which is an important feature that electron beam lithography and photo lithography do not possess.

In our research, we established many improvements via replica molding method: the flexible PDMS and the alignment layer that are integrated into an alignment substrate can 
avoid cracks that occur often in traditional polymer alignment layer during the bending processes; our method has increased the flexibility of rollable LC devices so that they might have more applications in flexible displays. Last but not least, the flexible LC device fabricated by this method was found to possess low driving voltage and comparable response time, which enables us to consume less power while maintaining better image fluency when we use it in displays.

\section{Experiments}

We used replica molding method to produce flexible LC devices. Replica molding method has been functioned to produce a wide range of structured surfaces such as diffraction gratings ${ }^{4)}$, compact disks ${ }^{5,6)}$ and micro devices ${ }^{7)}$. It includes three simple steps: dropping, curing and peeling. Figure 1 is the flowchart of the fabrication process. First, we use droppers to drop PDMS on the silicon mold. Then we bake PDMS at $90^{\circ} \mathrm{C}$ for 90 minutes in an oven. The PDMS can readily convert into solid elastomers by cross-linking. After it is cured, we peel PDMS against the rigid mold along the direction of grating. The formulation, application, and fabrication of PDMS elastomers have been studied extensively in Reference 8 .

What we've demonstrated here is the process of using replica molding method to fabricate flexible substrates. The mold is the silicon wafer with microgrooves patterned during traditional photo-lithography process. The wafer is covered with photoresist by spin coating. The photoresist-coated wafer is then baked to eliminate excess solvent. After baking, the photoresist is exposed to a pattern of ultraviolet light. The following chemical change allows some of the photoresist to be removed by developer. In the etching step, plasma helps to remove the uppermost layer of the substrate in the areas that are not protected by photoresist. Finally, the remained photoresist is removed from the substrate. The pattern of mold is a grating of $1 \mu \mathrm{m}$ pitch and the width of the line is $500 \mathrm{~nm}$. If the pitch is too larger, they will not have enough good ability to align LC molecules. The PDMS is commercially available from Dow Corning Corporation. It is supplied as a two-part kit: a liquid silicone rubber base and a curing agent. In order to function it properly, we mix the curing agent and liquid silicone rubber base at a ratio of 1:5. We have also mixed them at the ratio from 1:6 to 1:10; since that the results are almost the same, we can see that the ratio of mixture do not influence our outcome. The PDMS elastomer is isotropic and homogeneous with good thermal stability up to $186^{\circ} \mathrm{C}$. Substrates made up of this material can be deformed mechanically to fit the 
patterns and relief structures on the mold surfaces.

In order to make electrode layers, we use RF magnetron sputter to coat indium tin oxide (ITO) layers on the microgroove surface of the PDMS substrates. After that the two patterned PDMS substrates are arranged to be a $90^{\circ}$ twisted-nematic (TN) cell. The LC material is nematic LC MJ9915 offered by Chung-Hwa Picture Tube Company. After using uncured PDMS to seal the substrates, we insert cell into polarizer and analyzer (they are perpendicular to each other). And we use the $632.8 \mathrm{~nm}$ line He-Ne laser as the source to measure the electro-optical (EO) transmittance and the response time.

\section{Results and discussion}

Figure 2a shows the atomic force microscopy (AFM) image of grating nanostructures on a mold coated by photoresist in contact mode. Figure $2 \mathrm{~b}$ shows the AFM image of grating nanostructures on PDMS; we have prepared our sample by replicating from a silicon mold. The heights (peak to valley) of the grating nanostructures on the original mold are $55.7 \mathrm{~nm}$, while the heights of the PDMS grating nanostructures are $64.0 \mathrm{~nm}$. Because we've peeled PDMS off from the mold, the heights of the PDMS are slightly larger than those of the mold. This result indicate that using replica molding method to duplicate PDMS substrates for flexible displays is feasible because it can copy the groove as well as we wish or even better. In order to maintain cell gaps, we can also add the structure of spacer to the mold; therefore, there will be a spacer structure on the PDMS substrate to enhance the stability of cell gaps.

Figure 3 a shows the normally white state (the analyzer and polarizer are orthogonal) where a large percentage of the pattern is uniformly white. And figure $3 \mathrm{~b}$ shows the normally black state (the analyzer and polarizer are parallel) where cell is almost dark black. From figure 3, we can see that the PDMS substrates have great ability to align LC molecules just as conventional polyimide (PI) does._Figure 4 shows the normalized EO transmittance and the response time of our flexible LC cell fabricated by replica molding method in the flat state. The EO transmittance begins to decrease at about 1.5 volts in the normally white mode and the response time is about $24.1 \mathrm{~ms}$. The driving voltage $(1.5 \mathrm{~V})$ is as low as that of the conventional LCDs so that it can consume less power. In this research, since that ITO layers serving as conductive materials may not have sufficient flexibility to match the PDMS substrates, the use of conductive polymer substituting for ITOs in order to increase the degree of bending would be an important extended research target in the future. 


\section{Conclusions}

In conclusion, replica molding method is truly an efficient method to duplicate substrates for flexible displays. Via this method, we can successfully transfer the microgroove pattern from silicon mold to PDMS. The PDMS can readily convert into solid elastomers by cross-linking and the LC molecules can thus align immediately on the patterned PDMS substrates without coating additional polymer layers in order to align LCs. It is clear that we have combined the alignment-layers and substrates together successfully. They are both made by PDMS. Also, the flexible LC cell fabricated by this method has low driving voltage and comparable response time so that it can consume less power while possessing better image fluency. It is truly an important milestone for the soft flexible displays and its further applications.

\section{Acknowledgments}

We are grateful for Mr. W. L. Huang for his participation in earlier phase of this work. We acknowledge the support from the National Science Council, the Ministry of Education, the National Nano Device Laboratories, and the National Applied Research Laboratories of Taiwan. 


\section{References:}

1) S. Y. Chou, P. R. Krauss, and P. J. Renstrom: J. Vac. Sci. Technol. B14 (1996) 4129.

2) Y. T. Kim, S. Hwang, J. H. Hong, and S. D. Lee: Appl. Phys. Lett. 89 (2006) 173506.

3) Y. Xia and G. M. Whitesides: Annu. Rev. Mater. Sci. 28 (1998) 153.

4) M. C. Hutley: Diffraction Gratings (Academic, New York, 1982).

5) H. C. Haverkorn van Rijsewijk, P. E. J. Legierse, and G.E. Thomas: Philips Tech. Rev 40 (1982) 287.

6) B. D. Terris, H. J. Mamin, M. E. Best, J. A. Logan, D. Rugar, and S. A. Rishton: Appl. Phys. Lett. 69 (1996) 4262.

7) D. A. Kiewit: Rev. Sci. Instrum. 44 (1973) 1741.

8) Y. Xia, J. J. McClelland, R. Gupta, D. Qin, X. M. Zhao, L. L. Sohn, R. J. Celoua, and G. M. Whitesides: Adv. Mater. 9 (1997) 147. 


\section{Figure captions}

Figure 1. Flowchart of fabrication processes for PDMS substrates.
(a) Silicon mold.
(b) Dropping PDMS to mold.
(c) Baking PDMS at $90^{\circ} \mathrm{C}$ for 90 minutes.
(d) Peeling PDMS against mold.
(e) Flexible PDMS substrate.

Figure 2. AFM image of (a) Grating nanostructures on a mold (the heights are $55.7 \mathrm{~nm}$ ) and (b) Grating nanostructures on a PDMS produced by replication from the silicon mold (the heights are $64.0 \mathrm{~nm}$ ).

Figure 3. The cell of $90^{\circ}$ twisted-nematic LCs (PDMS substrates).
(a) Normally white state (the analyzer and polarizer are orthogonal).
(b) Normally black state (the analyzer and polarizer are parallel).

Figure 4. EO transmission and the response time of our LC cell with PDMS substrates.

(a) Normalized transmittance as a function of applied voltage.

(b) The falling and rising times, $\tau_{90-10}=24.1 \mathrm{~ms}$ and $\tau_{10-90}=52.3 \mathrm{~ms}$ under the applied voltage $13 \mathrm{~V}$ (1 kHz square waveform). 


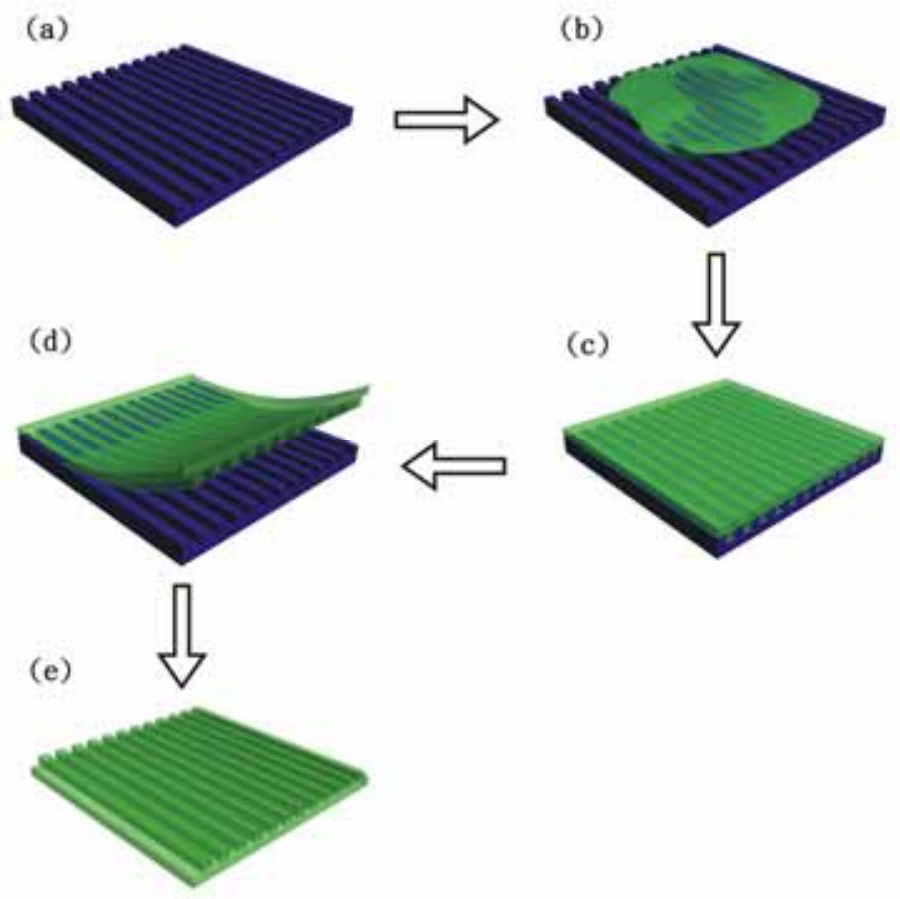

Figure 1

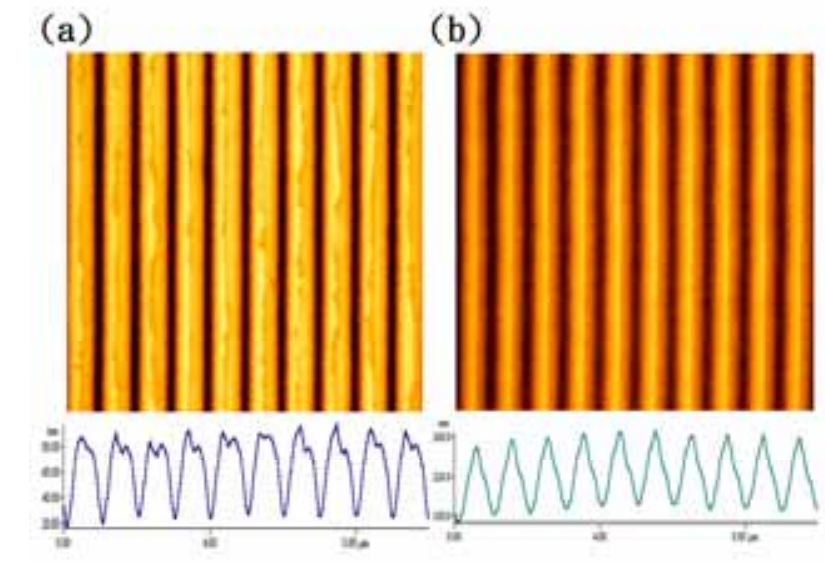

Figure 2

(a)

(b)
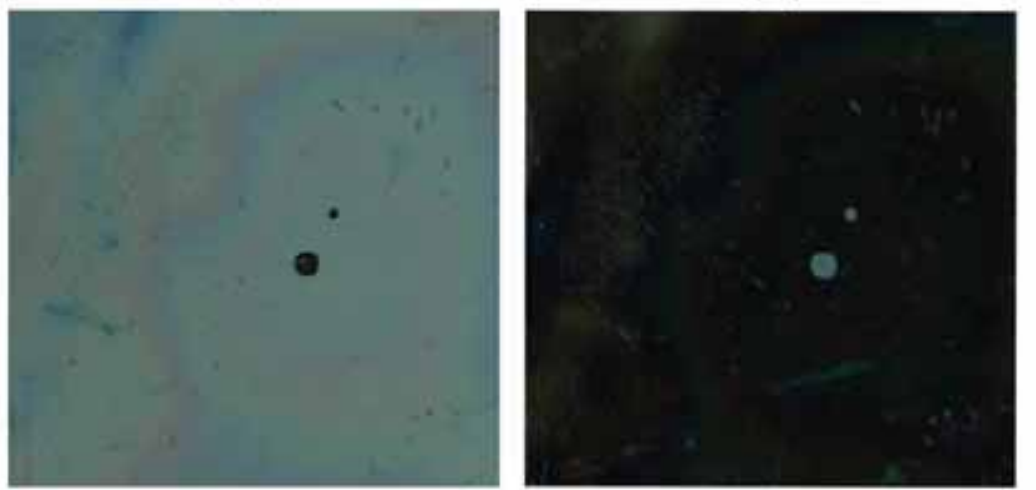

Figure 3 

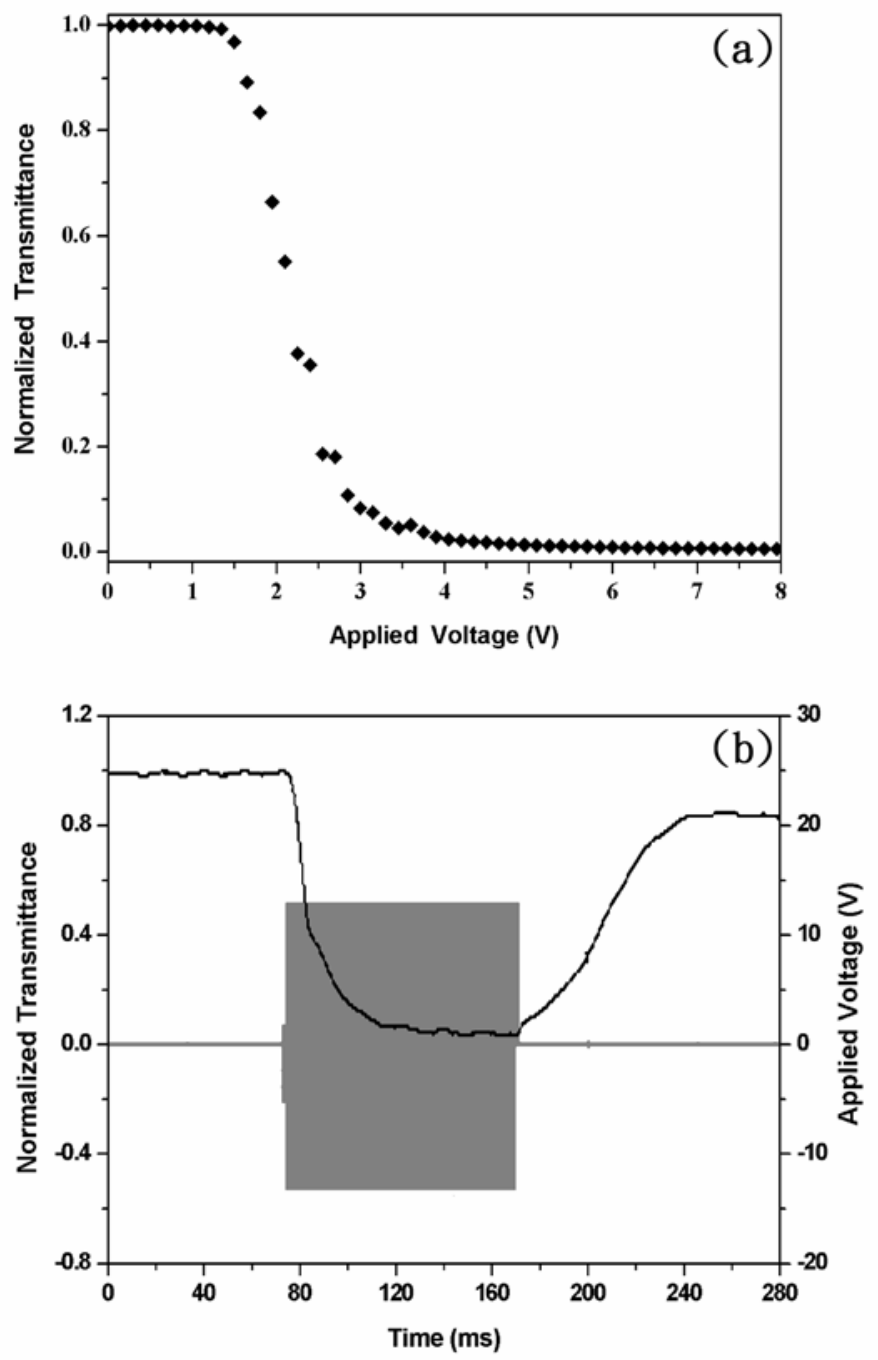

Figure 4 


\title{
Effect of insulating nanoparticles doping on electro-optical characteristics in nematic liquid crystal cells
}

\author{
Wei-Ting Chen, Pei-Shiang Chen, and Chih-Yu Chao \\ Department of Physics, National Taiwan University, Taipei 10617, Taiwan
}

In this paper, the transient currents and the voltage holding ratio (VHR) generated by an applied direct voltage have been measured in nematic liquid crystal (LC) cells. The experimental results show that doping insulating nanoparticles such as $\mathrm{ZnO}, \mathrm{TiO}_{2}$ and $\mathrm{Si}_{3} \mathrm{~N}_{4}$ in nematic LCs leads to a reduction in the moving-ion density in LC cells and drastically reduces the transient currents as well as threshold voltage. This doping method improves the electro-optical characteristics in nematic LC cells. Moreover, we find that the VHR values are maintained higher than 95\% in these doped LC cells. Such a high VHR observed in doped LC cells is a superior feature for future LC display applications. 


\section{INTRODUCTION}

Displaying information is essential in many aspects of our daily life. Among all display systems, liquid crystal display (LCD) is more than half of the total display markets on account of many advantages, including the flat panel, low weight, high definition, low driving voltages, and low power consumption. Currently, almost all LCDs are made up of nematic liquid crystal (LC) mixtures. And one of the most common modes for nematic LC displays is twisted nematic (TN) mode. Although the nematic LCs have shown great promise for use in a wide range of optical devices, the degrading tendency of the performance is undoubtedly a significant barrier for practical applications since people's requisition for display quality is getting higher.

The performance of a LC device is mainly affected by the alignment layers and the LC mixtures used. One of the most crucial causes of performance degradation for nearly all the LCDs is the ions within the LC devices. These ions may come from the LCs, the alignment layers, the surrounding glue, generated by UV polymerization, or during the filling process. When a voltage is applied across the LC cell, such ions begin to move and finally are adsorbed by the alignment layers. The adsorbed ionic layers, called the electric double layers ${ }^{1}$, create an inner dc field and decrease the effective voltage for the LC layer. This screening effect increases the threshold 
voltage $\left(\mathrm{V}_{\text {th }}\right)$ required for the display and gives rise to flicking as well as image sticking. In order to perform high-quality images, the LCs employed in a LCD must possess a sufficiently high resistivity and a reasonably low concentration of ion impurity. It is the reason why cyano compounds, known to have high conductivity and ion trapping near the alignment layers, such as E7 and 5CB, are not employed in the LCD industry.

Recently, considerable attention has been paid to the influence of ions on the electro-optical characteristics of LCDs. Some experiments have been done to explain the motion of charges in the LC cells, such as the studies on the transient currents ${ }^{2}$, measurements of the electro-optical properties ${ }^{3}$, and the simulation of the voltage holding ratio ${ }^{4}$. However, the voltage holding ratio (VHR) of the LC cells was not measured in these experiments.

Nevertheless, the VHR is an important performance parameter for an active matrix addressed LCD. Each pixel in LCD is driven by a thin-film transistor charging the pixels by signal pulses. The storing charges of a pixel should be maintained until the pixel is addressed in the next driving frame. If the capacity of keeping the charge at a pixel is not enough, a decrease in contrast, non-uniformity of the brightness or even image flickering can occur. The capability of keeping charges is called the VHR defined as the ratio of voltage at a pixel at the end of the frame time to the applied 
pulse voltage. For a high-end LCD the VHR must be high and uniform.

To improve the electro-optical behavior associated with the ion impurity, at least two primary approaches have been made. One is to modify the surface of LC cells ${ }^{5}$ by coating an ion trapping layer above the ITO electrode and the other is the structure modification of the LC host by incorporation with nanoscale materials ${ }^{6,7}$. Carbon nanotubes (CNTs) have long been used as guest dopants in nematic LCs owing to many of their unique physical, chemical and electronic properties. Some advantages were found in the CNTs doped LC cells such as the reduction of the residual $\mathrm{dc}^{7}$, which is related to the image sticking problems. But in our previous work ${ }^{8}$, we found that the CNTs doped cell can not maintain a high VHR value when the applied voltage is above the Freedericksz threshold. In this paper, we dope the insulating nanoparticles such as $\mathrm{ZnO}, \mathrm{TiO}_{2}$ and $\mathrm{Si}_{3} \mathrm{~N}_{4}$ in the nematic LC cells. Our experimental results show that the addition of insulating nanoparticles leads to a reduction of the transported ion concentration. Simultaneously, a high VHR value is observed.

\section{EXPERIMENTAL}

For cell fabrication, the nematic LC MJ9915 offered by Chung-Hwa Picture Tube Company was used. And the insulating nanoparticles are purchased from Nanostructured \& Amorphous Materials, Inc. In this work, we use three kinds of 
insulating nanoparticles $\mathrm{Si}_{3} \mathrm{~N}_{4}, \mathrm{ZnO}$ and $\mathrm{TiO}_{2}$ as guest dopants with diameters about 25 35 nm. Each empty LC cell was manufactured with two flat glass substrates coated with indium tin oxide (ITO) electrodes and polyimide alignment layers for planar orientation in a $90^{\circ} \mathrm{TN}$ configuration, where a cell gap of $4 \mu \mathrm{m}$ was ensured by spacers. The overlapped area of the electrodes was $1 \mathrm{~cm}^{2}$. The host-guest LC-nanoparticles mixtures were prepared by adding nanoparticles into LC MJ9915 in its isotropic phase. The mixtures were ultrasonicated and then stirred to promote dispersion. The mixtures with different kinds of nanoparticles and concentration were then introduced into empty cells by capillary action in the isotropic phase. A schematic illustration of the transient current measurement is shown in Fig. 1. For the measurements we apply a square voltage with amplitude $1.5 \mathrm{~V}_{\mathrm{pp}}$ and frequency $0.5 \mathrm{~Hz}$. The resulting leakage current is amplified and visualized on the scope. We repeat the waveforms several times to lower the noise by software averaging. To exclude the charging current caused by the reorientation of the LC molecules, we use voltages below the Freedericksz threshold. Because this charging current interferes with the ion current, voltages above threshold would destroy the reliability of the transient current measurements ${ }^{9}$. Transmittance of the TN LC cell from a He-Ne laser was also measured with a photo detector. All cells were measured by using a cross-polarizer configuration, yielding normally white (NW) mode, under an applied dc voltage. We 
use a $5 \mathrm{~V}$ dc pulse to measure the VHR. The detailed experimental method for VHR measurement can be found in Ref. 5.

\section{RESULTS AND DISCUSSION}

Figure 2 shows the transient current induced by the polarity-reversed voltage.

One have known that the charging current appears within a very short time after the polarity is reversed, followed by a transient current peak caused by the moving ions. We find that the addition of these three kinds of insulating nanoparticles $\left(\mathrm{ZnO}, \mathrm{TiO}_{2}\right.$, and $\mathrm{Si}_{3} \mathrm{~N}_{4}$ ) as dopants effectively reduce the transient current bumps. We think that the doped insulating nanoparticles have influenced the LC host properties, presumably due to sufficient ion trapping, and caused the amount of moving charges to decrease, thus reducing the transient current. For NW configuration, the threshold voltage $\left(\mathrm{V}_{\text {th }}\right)$ is defined as the voltage when the light transmittance is $90 \%$ of the initial transmittance at zero voltage. Figure 3 shows the transmittance of various TN cells, and Table I shows the corresponding $\mathrm{V}_{\text {th }}$ for doped and undoped cells. One can see that the $\mathrm{ZnO}$, $\mathrm{TiO}_{2}$, and $\mathrm{Si}_{3} \mathrm{~N}_{4}$ nanoparticles doped LC cells possess lower threshold voltage compared to that of undoped one. This phenomenon can also be explained by the guest dopants whose interaction with ion impurities made the screening effect less effective and allowed the LC molecules to experience a relatively larger effective 
voltage within the cell and thus lead to the reduction of the threshold voltage. Figure 4(a) and (b) is the conceptual depiction of ions moving in the LC cell with and without insulating nanoparticle doping. Figure 4(b) illustrates that the insulating nanoparticles can trap ions in the LC material and leads to fewer ions flowing to two sides of the cell and thus abates the screening effect. Table II shows the VHR values of doped and undoped TN LC (MJ9915) cells. In comparison with the CNTs doped cells ${ }^{8}$, the insulating nanoparticles doped cells possess a sufficiently high VHR. Because the nanoparticles are insulating, they will not increase the conductance of LCs. However, the conductance of the CNTs-doped cell will increase when the CNTs are reoriented from planar to homeotropic orientation by LC molecules above the Freedericksz threshold, resulting in lowering of $\mathrm{VHR}^{10}$.

\section{CONCLUSIONS}

In summary, the transient current and the voltage dependent transmittance in TN LC cells doped with $\mathrm{ZnO}, \mathrm{TiO}_{2}$, and $\mathrm{Si}_{3} \mathrm{~N}_{4}$ nanoparticles were studied. The lower transient current found in the doped cells indicates that doping insulating nanoparticles into the LC cell has the ability to decrease the moving-ion density, and thus suppresses the undesired field-screening effect and contributes to a reduction of the threshold voltage. The transmittance curve also confirmed that the residual dc 
caused by the ion impurity was reduced. We speculate that the ability to reduce the screening effect is due to the ion trapping by insulating nanoparticles which are polarized under an external electric field. Besides, the VHR of the doped cell can still be maintained higher than 95\% which indicates that these insulating nanoparticles will not decrease the resistivity of the LC cell. Therefore the insulating nanoparticles are better choices for one to use in order to avoid the disadvantages of LC devices caused by ion impurities.

\section{ACKNOWLEDGEMENTS}

We thank the Industrial Technology Research Institute for VHR measurements. This work was supported by the National Science Council and Ministry of Education of Taiwan. 


\section{REFERENCE}

${ }^{1}$ H. Mada and K. Osajima, J. Appl. Phys. 60, 3111 (1986).

${ }^{2}$ C. Colpaert, B. Maximus, and H. Pauwels, Proc. SID Res. Conf. 1993, 301.

${ }^{3}$ W. Lee, J. S. Gau, and H. Y. Chen, Appl. Phys. B: Lasers Opt. 81, 171 (2005).

${ }^{4}$ N. Sasaki, Jpn. J. Appl. Phys. 37, 6065 (1998).

${ }^{5}$ K. Furuichi, J. Xu, M. Inoue, H. Furuta, N. Yoshida, A. Tounai, Y. Tanaka, A.

Mochizuki, and S. Kobayashi, Jpn. J. Appl. Phys. 42, 4411 (2003).

${ }^{6}$ W. Lee, C. Y. Wang, and Y. C. Shih, Appl. Phys. Lett. 85, 513 (2004).

${ }^{7}$ I. S. Baik, J. Y. Lee, S. Y. Jeon, K. H. An, J. W. Choi, S. H. Lee, and Y. H. Lee, Appl. Phys. Lett. 87, 263110 (2005).

${ }^{8}$ P. S. Chen, C. C. Huang, Y. W. Liu, and C. Y Chao, Appl. Phys. Lett. 90, 211111 (2007).

${ }^{9}$ C. Colpaert, B. Maximus, and A. De Meyere, Liq. Cryst. 21, 133 (1996).

${ }^{10}$ I. Dierking and S. E. San, Appl. Phys. Lett. 87, 233507 (2005). 


\section{TABLE}

I. Threshold voltage of doped and undoped TN cells.

\begin{tabular}{lc}
\hline \hline & $\mathrm{V}_{\text {th }}(\mathrm{V})$ \\
\hline Undoped & 1.79 \\
$0.02 \% \mathrm{ZnO} / \mathrm{TiO}_{2} / \mathrm{Si}_{3} \mathrm{~N}_{4}$-doped & $1.7 / 1.67 / 1.69$ \\
$0.04 \% \mathrm{ZnO} / \mathrm{TiO}_{2} / \mathrm{Si}_{3} \mathrm{~N}_{4}$-doped & $1.63 / 1.64 / 1.61$ \\
$0.06 \% \mathrm{ZnO} / \mathrm{TiO}_{2} / \mathrm{Si}_{3} \mathrm{~N}_{4}$-doped & $1.6 / 1.63 / 1.54$ \\
\hline
\end{tabular}

II. Voltage holding ratio of doped and undoped TN cells.

\begin{tabular}{lll}
\hline & & VHR (\%) \\
\hline & & 97.22 \\
$0.06 \%$ & $\mathrm{ZnO}$-doped & 96.83 \\
$0.06 \%$ & $\mathrm{TiO}_{2}$-doped & 95.69 \\
$0.06 \%$ & $\mathrm{Si}_{3} \mathrm{~N}_{4}$-doped & 97.71 \\
\hline
\end{tabular}




\section{FIGURE CAPTIONS}

FIG. 1 Schematic illustration of the setup for the transient current measurement.

FIG. 2 Transient currents of (a) $\mathrm{ZnO}$, (b) $\mathrm{TiO}_{2}$, and (c) $\mathrm{Si}_{3} \mathrm{~N}_{4}$ doped LC cells.

FIG. 3 Transmittance through (a) $\mathrm{ZnO}$, (b) $\mathrm{TiO}_{2}$, and (c) $\mathrm{Si}_{3} \mathrm{~N}_{4}$ doped LC cells under an applied dc voltage.

FIG. 4 Conceptual depiction of ions moving in the cell (a) without, and (b) with doping insulating nanoparticles.

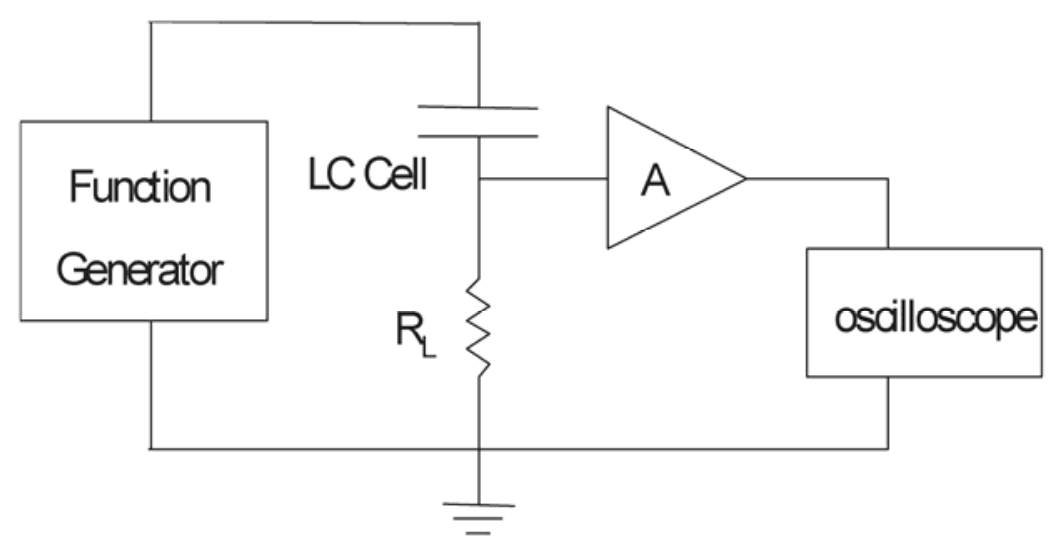

FIG.1 

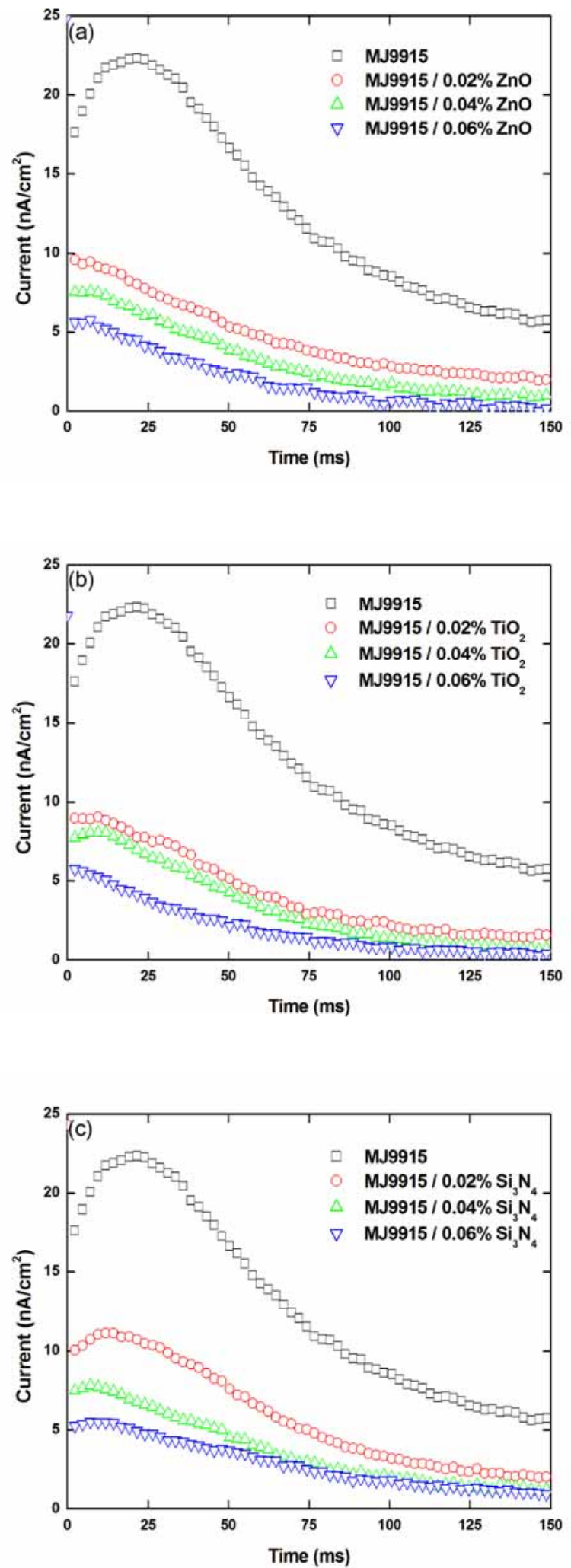

FIG. 2 

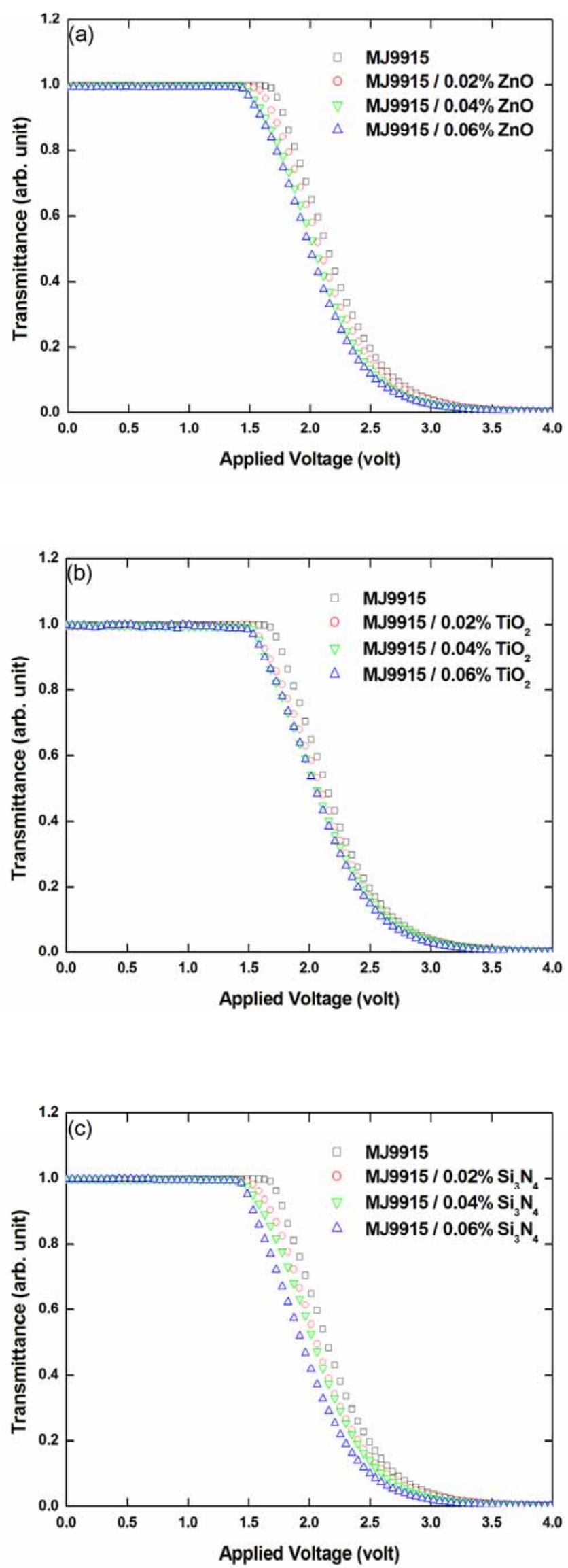

FIG. 3 

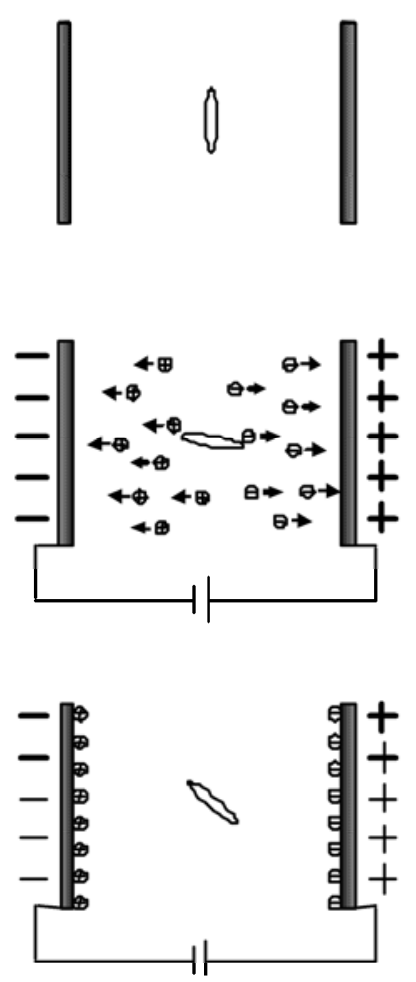

(a)
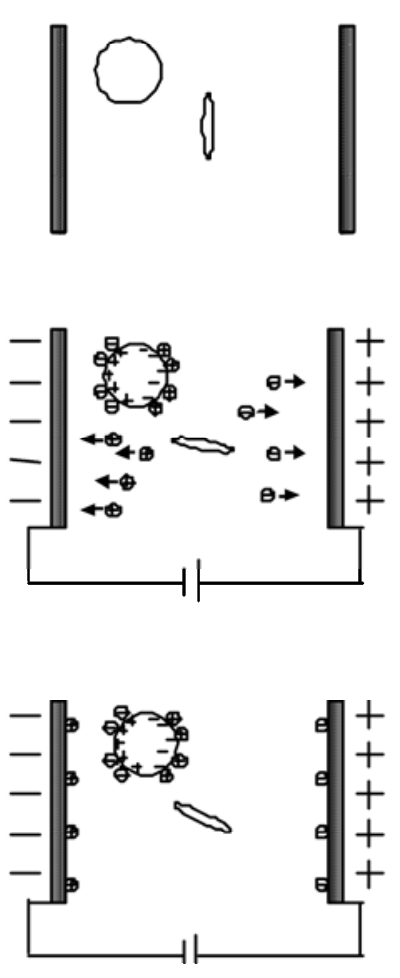

(b)

FIG. 4 